\title{
Effect of specimen bed on the material removal due to the repetitive single ball impacts
}

\author{
Mehdi Akhondizadeh ${ }^{*}$ and Meysam Atashafrooz \\ Mechanical Engineering Department, Sirjan University of Technology, Sirjan, Iran
}

Received: 4 April 2017 / Accepted: 29 May 2019

\begin{abstract}
Specimen wear due to the repetitive single ball impact is investigated experimentally by a drop test machine. Effect of the impact energy, incidence angle and bed material is studied. Specimen mass loss is measured after 1000 impacts. The crater dimension on the specimen surface is measured to indicate its correlation with the wear variation. Results show that the rubber bed has the undeniable positive role in decrement of the wear due to impact comparing with the steel bed. A relation between the energies which give the same wear, in both cases of the rubber and steel bed, is extracted. Results can be helpful in designing the appropriate bed where the medium and high energy impacts encouraged.
\end{abstract}

Keywords: Impact / wear / rubber bed / steel bed / impact energy

\section{Introduction}

Impact wear has been defined as the wear of a solid surface due to the repetitive exposure to dynamic contact by another body [1]. The definition distinguishes impact wear from erosive wear which occurs by impact of streams of small particles. Impact wear includes the large body collisions which the influencing parameters can be controlled whereas; the eroding particles are small and randomly impact the target surface. Many industrial situations involve impacting bodies in which failures due to wear can be costly. Such events occur in tumbling mills [2], silo outlet, belt conveyors, etc. The cost concerns associated not only with the need of frequent part replacement, but also with the down time occurred. The first studies of the impact wear have been carried out by Engle [3]. There are several experimental researches at initial years of study [4-6]. There are several works in recent years. Veinthal et al. [7] carried out the surface fatigue wear testing by the abrasive impact wear (AIW) tester. They studied the relation between high energy impact wear behavior and the surface fatigue wear of $\mathrm{Fe}-\mathrm{Cr}-\mathrm{C}$ produced by different cooling conditions. Researchers show that the maximum impact wear occurs at the incidence angles about $25-30^{\circ}$ [8-11]. The impact wear tester includes the hammer type machines $[12,13]$ that may have deviations of the free impacts occur in some cases like tumbling mills [14] and conveyor belt chutes. Some of the impact testers simulate the low impact energies $[9,11]$ which is not applicable for the high impact energy issues.

\footnotetext{
* e-mail: m.akhondizadeh@sirjantech.ac.ir
}

In the present work, the high energy free ball impacts, like that happen in many industrial cases are modeled in a drop test machine. The aim is to measure the effect of influencing parameters on the material removal due to repetitive single ball impacts. The most important purpose is to determine the effect of rubber bed, under the specimen, on the wear value. The main question is whether or not inserting the elastic elements under the impacted targets decrease the devastating effects of impact especially specimen wear. Results will be helpful in design of the proper bed to enhance the target wear behavior through the high impact energy impacts.

\section{Experimental set up}

\subsection{Drop test machine}

Experimental apparatus, as illustrated in Figure 1, is drop test machine in which ball is freely released of the specified height with the zero initial velocity and impacts the target specimen. The velocity is evaluated by the energy conservation law:

$$
\frac{1}{2} m v^{2}=m g h .
$$

Maximum height that can be achieved by the present machine is $2 \mathrm{~m}$. To have the little deviation of the impact velocity during the specified number of impacts, balls should be released of the same height. For this aim, a tapered duct, fixed at the specified height, is employed. Since the balls slowly pushed through the duct by a screw, they fall from the same 


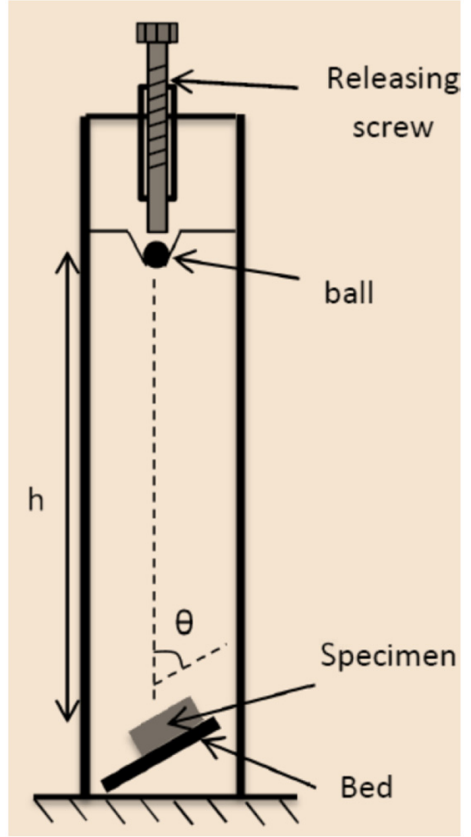

Fig. 1. Impact wear test machine.

height with the absolutely zero initial velocity. The specimen angular position is set by the tightening screws and is checked repeatedly. Two steel arms, covered by the plastic tape protecting specimen scratch, are used to compactly retain the specimen and bed on the base plate. After ensuring the target specimen is placed tightly, the experiments start until the specified number of impact is achieved. At the end, the specimen is washed smoothly to be cleaned of dust, moisture and debris for weighing. Balls are made of AISI 52100, (C 1\%, Mn $0.3 \%$, P $0.02 \%$, S $0.02 \%$, Si $0.2 \%$, Cr $1.2 \%$ ) with $700 \mathrm{HB}$ hardness. There is no regular manner for ball falling.

\subsection{Sample preparation}

Specimens are carbon steel (C 0.3\%, Mn 0.83\%, Si 0.82\%, P $0.11 \%$ ) with a hardness $77 \mathrm{HB}$ and are weighed by means of a $200 \mathrm{~g}$ balance with precise of 0.1 milligram. They are finished by soft emery polishing to be cleaned of any dust and debris. After this, they are completely washed to remove oil and micro-particles. After the specimens are dried, they are weighed and are ready to be tested. Washing, drying and weighing is repeated after the experiment to determine the material removal due to the impact.

\subsection{Specimen bed}

In order to investigate the effect of the bed on wear, a single layer of two different materials is placed between the specimen and base. The base plate is made of concrete to have a maximum rigidity. The bed is adhered on the concrete base by means of industrial glue. The glue layer is accurately remained thin to have a minimum influence on results. The steel and natural rubber, with a thickness of $5 \mathrm{~mm}$, which mechanical properties are given in Table 1 , are used for this aim.
Table 1. Mechanical properties of the rubber and steel bed.

\begin{tabular}{lllc}
\hline & $\begin{array}{c}\text { Density } \\
\left(\mathrm{Kg} / \mathrm{m}^{3}\right)\end{array}$ & $\begin{array}{c}\text { Modulus of } \\
\text { elasticity }(\mathrm{GPa})\end{array}$ & $\begin{array}{c}\text { Yield strength } \\
(\mathrm{MPa})\end{array}$ \\
\hline Steel & 7830 & 200 & 294 \\
Rubber & 1520 & 0.02 & 1.5 \\
\hline
\end{tabular}

\section{Results and discussion}

The impact behavior depends on the impact conditions such as impact velocity, coefficient of friction and impact angle. At the small impact angles, the major part of impact duration includes sliding while at the higher impact angles the rolling and rebound occurred. Since several number of impacts is required to have a measurable wear it is practically impossible to repeat all tests. However, tests 1 and 9 in Table 2 have been performed two times and the resultant error was $\pm 8 \%$, which is acceptable for the multi-variable dependent phenomenon as impact wear. Thirteen experiments with conditions given in Table 2 are performed. The variable impact parameters are the ball velocity, ball size, impact angle and specimen bed. Each of them includes 1000 impacts. The impact angle is the angle between the ball velocity direction and the specimen surface.

The effect of impact angle on specimen wear depends on ductility and brittleness of material [8-10]. The present results indicated that for the present specimens the wear maximizes at the impact angle of $30^{\circ}$. Wear occurs due to sliding in low angle impacts and due to the fatigue wear in high impact angles. It can be said that the impact wear is due to compound sliding and fatigue. The plastic craters on specimen surface are illustrated in Figure 2. Moreover, surface crack initiation and propagation occurs in brittle materials. The present specimens are ductile steels and the main viewed evidences are plastic craters compound with sliding.

Being elastic enough, the bed permits the specimen to move downward through the impact. By the way, a portion of energy is transmitted to the bed through the specimen and so the impact consequences on the surface, crater dimension and wear value, will be lowered. In contrast of this, a nearly rigid bed makes the specimen to be forged between the ball and bed and the failure occurs faster than in case of the elastic bed [15-17]. Wear results in case of $5 \mathrm{~mm}$ thick natural rubber and steel bed which are illustrated in Figure 3 reveal that the impact wear of specimen on the steel bed is about 3 times more than that of the rubber bed.

A relation between the wear and the impact energy is extracted by curve fitting in Excel. Fitting several relations on the obtained data determined that the power relation gives the best accordance. The obtained power relation is:

$$
w=k E^{n} .
$$

In which $w$ is the wear in milligram, $E$ is the impact energy in joule and $k$ and $n$ are factors given in Table 3. 
Table 2. Impact parameters and wear results.

\begin{tabular}{llllllll}
\hline Test No. & Bed & Velocity $(\mathrm{m} / \mathrm{s})$ & Ball Dia. $(\mathrm{mm})$ & Imp. Angle $(\mathrm{deg})$ & Impact energy & Initial weight $(\mathrm{g})$ & Wear $(\mathrm{mg})$ \\
\hline 1 & Rubber & 4 & 25 & 60 & 0.512213 & 118.3600 & 2.4 \\
2 & Rubber & 4 & 25 & 15 & 0.512213 & 120.4681 & 1.8 \\
3 & Rubber & 5.4 & 25 & 15 & 0.933507 & 104.7214 & 3.6 \\
4 & Rubber & 4 & 25 & 30 & 0.512213 & 114.879 & 3 \\
5 & Rubber & 4 & 20 & 30 & 0.262253 & 126.9132 & 1.2 \\
6 & Rubber & 4 & 30 & 30 & 0.885103 & 116.2029 & 3.6 \\
7 & Rubber & 5.4 & 25 & 30 & 0.933507 & 114.8787 & 3.6 \\
8 & Rubber & 6.25 & 25 & 30 & 1.250519 & 104.7211 & 4.8 \\
9 & Steel & 4 & 25 & 30 & 0.512213 & 116.2022 & 10.8 \\
10 & Steel & 5.4 & 25 & 30 & 0.933507 & 114.8784 & 13.2 \\
11 & Steel & 4 & 20 & 30 & 0.262253 & 104.7206 & 6 \\
12 & Steel & 4 & 30 & 30 & 0.885103 & 118.3596 & 12 \\
13 & Steel & 6.25 & 30 & 30 & 2.160896 & 120.4680 & 16.8 \\
\hline
\end{tabular}

a)
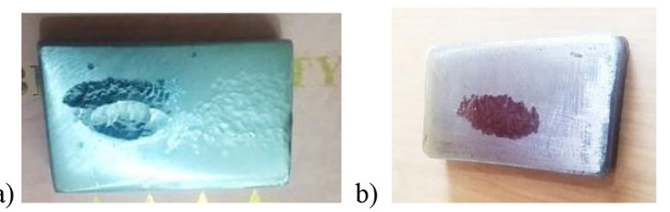

Fig. 2. Specimen surface at the end of test at the impact angle of (a) $90^{\circ}$ and (b) $15^{\circ}$.

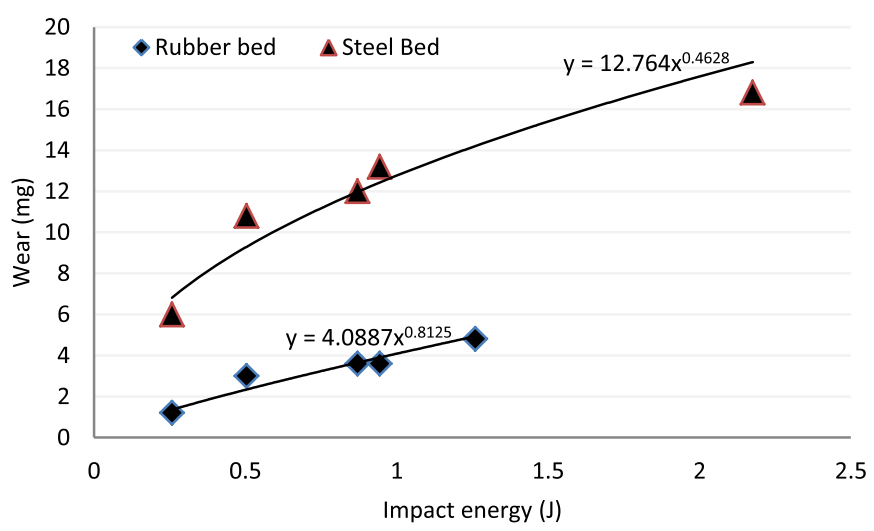

Fig. 3. Variation of the impact wear versus the impact energy, impact angle $30^{\circ}$.

Equaling the wear relations in form of equation (2) gives the ratio of impact energies in cases of the steel and rubber bed which give the same wear.

$$
w_{s}=w_{R} \frac{\overrightarrow{E_{s}}}{E_{R}}=0.085 E_{R}^{0.75} .
$$

In which $E_{R}$ and $E_{s}$ are the impact energies in case where the rubber and steel bed respectively which give the
Table 3. Factor of relation between wear and impact energy.

\begin{tabular}{lcc}
\hline & $K$ & $n$ \\
\hline Rubber bed & 4.0887 & 0.8125 \\
Steel bed & 12.764 & 0.4628 \\
\hline
\end{tabular}

same wear. $w_{R}$ and $w_{s}$ are the wear in case of the rubber and steel bed, respectively. The energy absorbed by the rubber bed, based on equation (2), can be evaluated as:

$$
\Delta E=100 \times \frac{E_{R}-E_{s}}{E_{R}} .
$$

Variation of the energy absorption as the percent of total energy is illustrated in Figure 4. A detailed study about the energy absorption in case of rubber and steel bed, including a layer of fluid on the target surface, has been presented in another publication [17]. Here, it can be seen that more than $80 \%$ of the impact energy is absorbed by the rubber bed. It means, for example, as illustrated in Figure 3 to have a wear about $6 \mathrm{mg}$ in case of rubber bed the impact energy about $1.5 \mathrm{~J}$ is required whereas, in case of steel bed about $0.25 \mathrm{~J}$ impact energy will be sufficient. It says that about $100 \times \frac{1.5-0.25}{1.5}=83 \%$ of impact energy has been absorbed by rubber bed. As the impact energy increases the energy absorption decreases. The reason is that in the high energy impacts there is not enough time for the specimen to move downwards and transmits energy to the bed by this means. As the impact energy increases, the rubber bed tends to behave in a rigid manner.

The effect of rubber thickness on impact wear is shown by using a thicker rubber bed. According to Figure 5 , wear in case of a $10 \mathrm{~mm}$ thick rubber bed is two third of the $5 \mathrm{~mm}$ thick rubber bed. It means that a higher energy is absorbed by increasing the rubber thickness. It may seem an obvious 


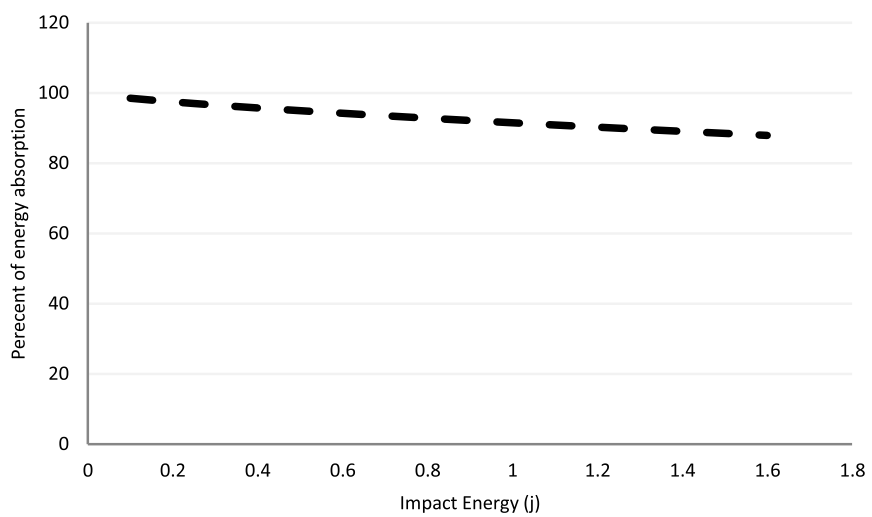

Fig. 4. Variation of the energy absorption by rubber bed versus the impact energy.

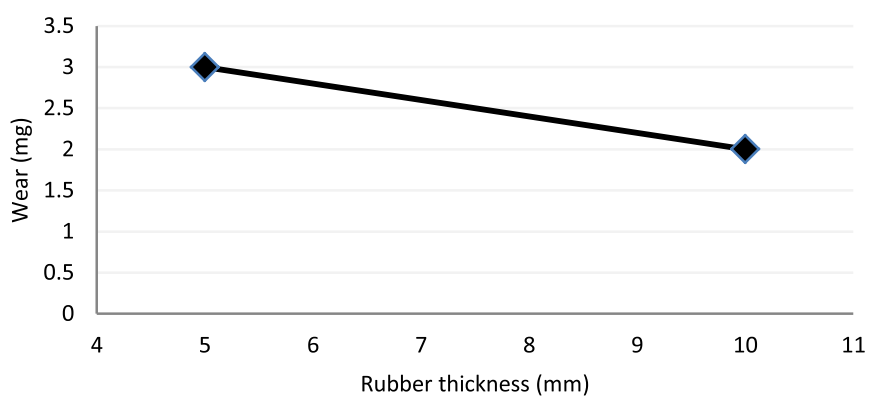

Fig. 5. Wear versus the rubber thickness.

result but the present data show that the relation between the bed thickness and wear behavior improvement is not linear. The experiments of Figure 5 have been repeated twice and an average of data is given in this figure. However, there are several sources of energy dissipation discussed in detail by Antonyuk et al. [18], but the only difference between the tests of Figure 5 was the rubber bed thickness. It was tried possibly to retain the other parameters unchanged. So it can be said that the only parameter which influences on the wear value or decreases it, is the rubber bed thickness.

Wear per impact energy is an important parameter in impact wear discussions [19]. It reveals the relative wear manner instead of the wear manner individually. However, the wear increases by increasing the impact energy but the relative wear tends to decrease as illustrated in Figure 6. This behavior is not clearly evident for rubber bed in this figure because of the selected ordinate range.

However, a general relation between the wear and impact parameters can rarely be found for prediction in all wear issues encountered but the present experimental data can be fairly estimated by the following relation.

$$
w=K_{w} v^{\alpha} r^{\beta}
$$

In which $w$ is the wear in milligram, $v$ is the impact velocity in $\mathrm{m} / \mathrm{s}, r$ is the ball radius in millimeter and $K_{w}, \alpha$ and $\beta$ are parameters fitted to the experimental data. The parameters which give the best estimation of the present experimental data are given in Table 4.

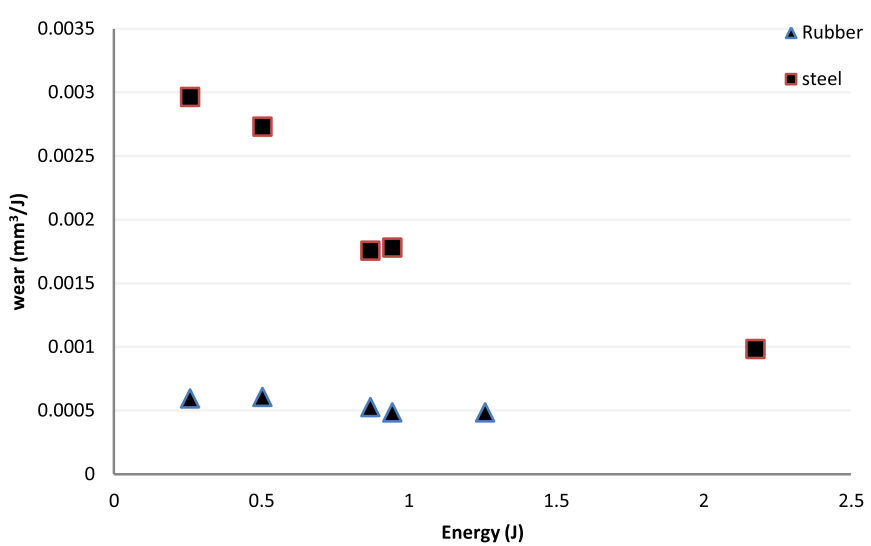

Fig. 6. Variation of the wear per energy versus the impact energy.

Table 4. Parameters of equation (5).

\begin{tabular}{lclc}
\hline & $K_{w}$ & $\alpha$ & $\beta$ \\
\hline Rubber bed & 0.0004 & 1.42 & 2.72 \\
Steel bed & 0.0014 & 1.3 & 2.72 \\
\hline
\end{tabular}

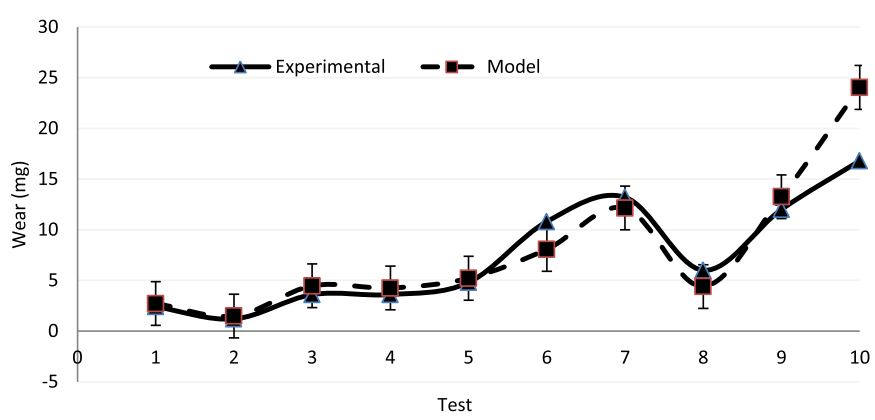

Fig. 7. Comparison of the experimental and predicted wear results.

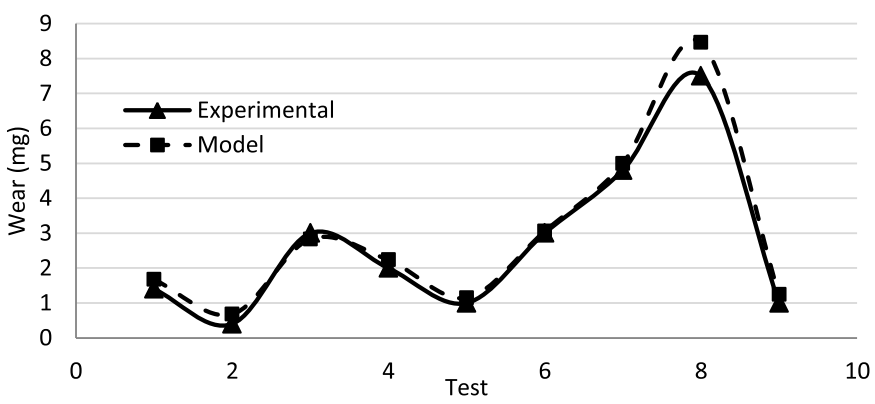

Fig. 8. Comparison of wear evaluated by equation (5) and experimental data of reference [20].

The comparison of the experimental data with the estimations of equation (5) is illustrated in Figure 7.

Moreover, the wear evaluations by equation (5) have been compared with the experimental data given by reference [20]. Results are illustrated in Figure 8. The 

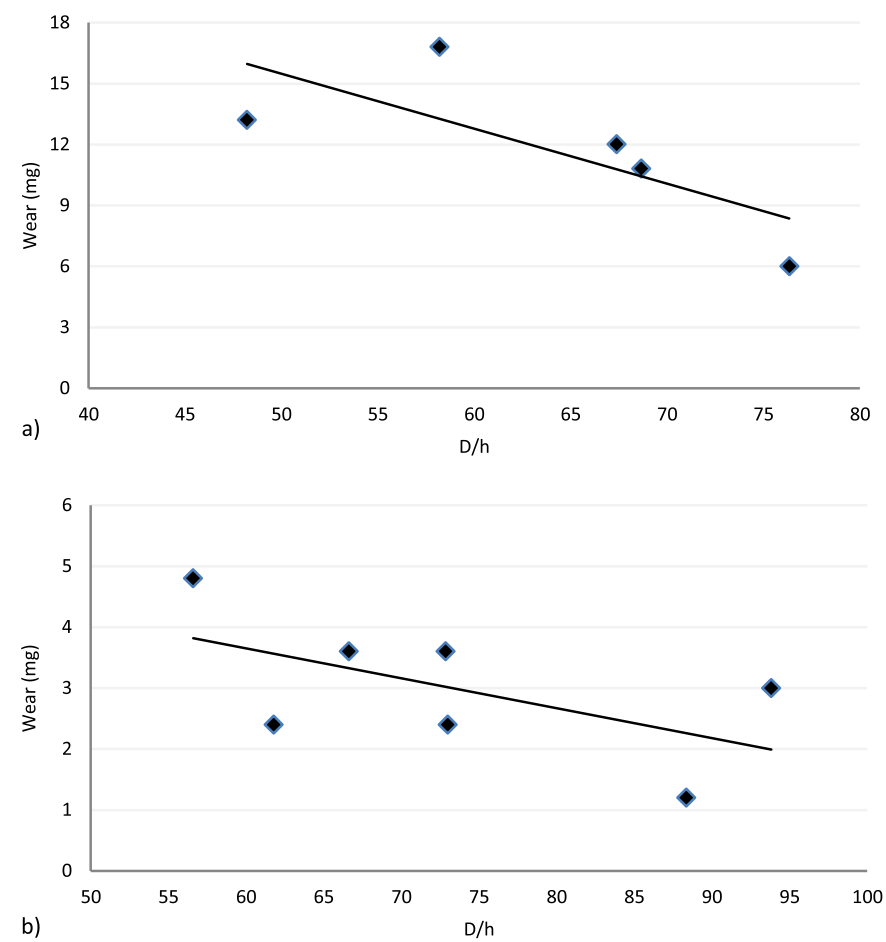

Fig. 9. Variation of the wear versus the ratio of the crater diameter to the crater depth. (a) Steel bed; (b) rubber bed.

evaluations were done by $K_{w}=0.00055, \alpha=1.3$ and $\beta=2.72$. It is interesting that the factors $\alpha$ and $\beta$ are as those obtained for steel bed.

Along with the impact wear, the crater dimension may be a sign of the impact intensity. The ratio of the crater diameter to its depth $(D / h)$ is considered as a parameter [19]. The variation of wear with respect to this characteristic parameter is illustrated in Figure 9. Results show that in the both cases of the rubber and steel bed increase of $D / h$ corresponds to the decrease of the impact wear. In the abrasion wear tests in pin-disk experiments, the normal force and sliding distance are two important parameters. In the impact wear experiments the crater depth is an indication of the normal force during contact and crater diameter indicates the sliding distance on target surface. The results say that the impact force has more influence on the impact wear value than the sliding in the present experiments.

It is confirmed by the present results that the impact wear highly depends on the impact energy $[8,19]$ and impact angle $[9,10]$. The initial impact energy is given as follows:

$$
T=\frac{1}{2} m v^{2}=\frac{1}{12} \pi \rho d^{3} v^{2} .
$$

In which $T$ is the kinetic energy, $\rho$ is ball density, $d$ is the ball diameter and $v$ is the ball impact velocity. Since the impact wear depends on the impact energy, it seems to be more dependent on the ball size than the ball velocity. The present experimental data confirm this, as

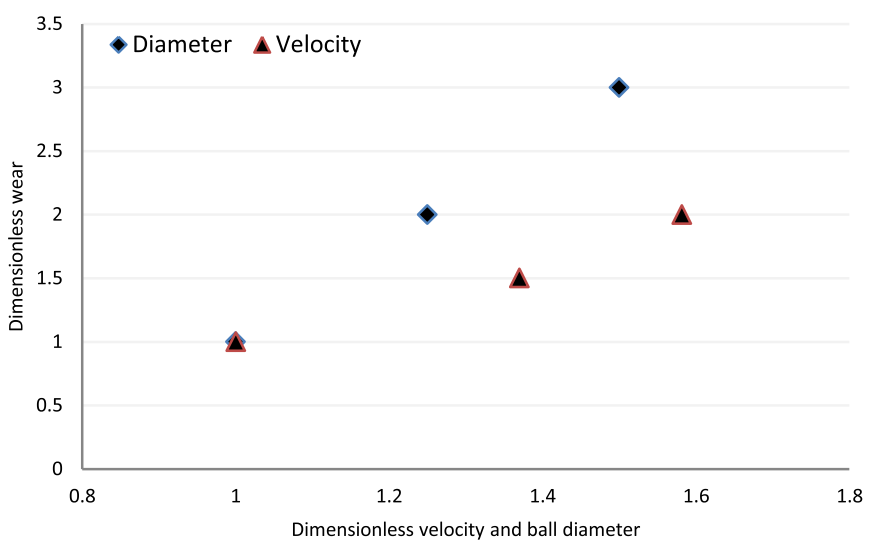

Fig. 10. Variation of wear versus the dimensionless ball velocity $\left(v / v_{\min }\right)$ and ball diameter $\left(d / d_{\min }\right)$.

Table 5. Ratio of big to small crater diameter, Impact angle $30^{\circ}$, ball size $25 \mathrm{~mm}$, steel bed.

\begin{tabular}{llll}
\hline Imp. velocity $(\mathrm{m} / \mathrm{s})$ & 4 & 5.42 & 6.26 \\
Ratio $\left(D_{\max } / D_{\min }\right)$ & 1.024 & 1.19 & 2 \\
\hline
\end{tabular}

illustrated in Figure 10. Elliptical craters are generated on the specimen surface due to the inclined impacts [20]. The ratio of the larger diameter to the smaller diameter may be a sign of the sliding manner. The values of this ratio are given in Table 5 . According to the data of Table 5, this ratio increases as the impact velocity increases in the same manner as the impact wear. It means that the high impact velocity creates longer craters with more sliding distance.

\section{Conclusions}

A drop test machine is employed to study the effect of impact parameters on the impact wear. The wear due to impact is lowered noticeably in case of a rubber bed. Indeed it is shown that this type of bed absorbs about $80 \%$ of the impact energy and accommodates the wear. The relations obtained by curve fitting are used to evaluate the energy absorbed by the rubber bed. A relation between wear and impact parameters is proposed which fairly estimates the present wear data.

\section{References}

[1] A.W.L. De Gee, C.P.L. Commissaris, J.H. Zaat, The wear of sintered aluminium powder (sap) under conditions of vibrational contact, Wear 7 (1964) 535-550

[2] M. Akhondizadeh, M.F. Mahani, M. Rezaeizadeh, S.H. Mansouri, A new procedure of impact wear evaluation of mill liner (research note), Int. J. Eng.-Trans. A: Basics 28 (2015) 593

[3] P.A. Engel, Impact wear of materials, Elsevier, Amsterdam, Netherland, 1978 
[4] R.G. Bayer, P.A. Engel, J.L. Sirico, Impact wear testing machine, Wear 19 (1972) 343-354

[5] P.A. Engel, Percussive impact wear: A study of repetitively impacting solid components in engineering, Tribol. Int. 11 (1978) 169-176

[6] P.A. Engel, J.L. Sirico, Impact wear study of lubricated contacts, Asle Trans. 18 (1975) 279-289

[7] R. Veinthal, F. Sergejev, A. Zikin, R. Tarbe, J. Hornung, Abrasive impact wear and surface fatigue wear behaviour of Fe-Cr-C PTA overlays, Wear 301 (2013) 102-108

[8] H. Ashrafizadeh, F. Ashrafizadeh, A numerical 3D simulation for prediction of wear caused by solid particle impact, Wear 276 (2012) 75-84

[9] E. Rigaud, A. Le Bot, Influence of incidence angle on wear induced by sliding impacts, Wear 307 (2013) 68-74

[10] M. Akhondizadeh, M.F. Mahani, M. Rezaeizadeh, S.H. Mansouri, Experimental investigation of the impact wear, Mechanics \& Industry 15(1) (2014) 39-44

[11] T. Souilliart, E. Rigaud, A. Le Bot, C. Phalippou, Energybased wear law for oblique impacts in dry environment, Tribol. Int. 105 (2017) 241-249

[12] R. Lewis, A modelling technique for predicting compound impact wear, Wear 262(11) (2007) 1516-1521

[13] R. Tarbe, P. Kulu, Abrasive impact wear: Tester, wear and grindability studies, Doctoral Dissertation, Tallinn University of Technology, Estonia, 2009
[14] M. Akhondizadeh, M.F. Mahani, M. Rezaeizadeh, S.H. Mansouri, Prediction of tumbling mill liner wear: Abrasion and impact effects, Proc. Inst. Mech. Eng., Part J: J. Eng. Tribol. (2016), doi: 10.1177/1350650116635424

[15] S. Aman, P. Mueller, J. Tomas, S. Kozhar, M. Dosta, S. Heinrich, S. Antonyuk, Combined viscoelastic and elastic wave dissipation mechanism at low velocity impact, Adv. Powder Technol. 27 (2016) 1244-1250

[16] S. Pupeschi, R. Knitter, M. Kamlah, Y. Gan, Numerical and experimental characterization of ceramic pebble beds under cycling mechanical loading, Fusion Eng. Des. 112 (2016) $162-168$

[17] M. Akhondizadeh, M. Mahdavinasab, Energy dissipation in single ball impact on a fluid-layered specimen with the rubber and steel bed, Ind. Lubr. Tribol. 70 (2018) 1209-1216

[18] S. Antonyuk, S. Heinrich, N.G. Deen, J.A.M. Kuipers, Influence of liquid layers on energy absorption during particle impact, Particuology 7 (2009) 245-259

[19] M. Lindroos, M. Apostol, V.-T. Kuokkala, A. Laukkanen, K. Valtonen, K. Holmberg, O. Oja, Experimental study on the behavior of wear resistant steels under high velocity single particle impacts, Int. J. Impact Eng. (2015), doi: 10.1016/j. ijimpeng.2014.12.002

[20] M. Akhondizadeh, M. Fooladi Mahani, M. Rezaeizadeh, S.H. Mansouri, Theoretical and experimental modeling of impact wear, Ind. Lubr. Tribol. 70(3) (2018) 490-498

Cite this article as: M. Akhondizadeh, M. Atashafrooz, Effect of specimen bed on the material removal due to the repetitive single ball impacts, Mechanics \& Industry 20, 401 (2019) 